\title{
Colto e acculturato sono sinonimi?
}

\section{Claudio lacobini}

PUBBLICATO: 05 FEBBRAIO 2021

\section{Quesito:}

Sono molti i lettori che ci rivolgono domande a proposito di acculturato, mettendolo spesso in relazione con colto.

\section{Colto e acculturato sono sinonimi?}

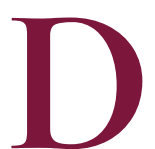

ovendo dare una risposta secca alla domanda se colto e acculturato sono sinonimi, dovremmo dire di no. Come sinonimo di colto, si può usare istruito, dotato di cultura, in certi contesti anche dotto, sapiente, preparato, mentre l'aggettivo acculturato è strettamente connesso al sostantivo acculturazione, un termine che ha origine negli studi antropologici per indicare un processo di mutamento della cultura e delle abitudini sociali di un popolo non per cause interne o spontanee, ma per l'influenza dovuta a contatto con una cultura diversa a cui viene riconosciuto maggiore potere o prestigio.

Il termine acculturazione è stato elaborato alla fine del diciannovesimo secolo dagli studiosi di antropologia statunitensi in riferimento ai profondi cambiamenti nelle abitudini e nella cultura degli Indiani d'America in seguito al contatto forzoso con la civiltà occidentale. Il concetto di acculturazione, oltre ai processi culturali conseguenti alla conquista violenta di un territorio e all'assoggettamento delle popolazioni che vi vivono, è stato poi esteso, nella letteratura antropologica e sociologica, a diversi altri tipi di contatto culturale che possono produrre cambiamenti notevoli negli usi, costumi, credenze di una popolazione: in primo luogo all'assimilazione di tratti culturali in seguito a fenomeni migratori, o al contatto tra popolazioni contigue caratterizzate da diverso potere economico e sviluppo tecnologico.

L'assimilazione delle caratteristiche comportamentali di una determinata cultura o di un gruppo di persone a cui un individuo appartiene, come ad esempio l'acquisizione spontanea di una lingua da parte di un infante, è definita con il termine inculturazione, o anche con il sinonimo socializzazione. Con deculturazione viene invece definito il processo di perdita delle caratteristiche culturali tipiche di un popolo, normalmente a seguito di un contrapposto processo di acculturazione. Tutti questi termini stentano però a essere impiegati al di fuori dell'ambito delle discipline sociologiche e antropologiche; inculturazione è impiegato anche nella cultura cristiana per indicare l'assimilazione all'interno delle pratiche e dei precetti religiosi di elementi culturali provenienti da altre tradizioni, come, ad esempio, dalla tradizione classica greco-latina.

Il verbo acculturarsi insieme alla forma aggettivale del participio passato, oltre che in riferimento al concetto antropologico e sociologico di acculturazione, ha visto recentemente estendere il proprio ambito semantico al più generico significato di acquisire (o cercare di acquisire) una cultura raccogliticcia, superficiale, spesso velleitaria. Tra gli esempi di questo uso che si possono ricavare dal web:

- Per capire se parlando di spazio e terra chi scrive ha conoscenza di astronomia, geografia oppure si è acculturato sul web. 
- con tutta la petulanza di chi é acculturato

- uno degli amati reality show con cui si è acculturato

- Ricchi, giovani e acculturati: chi sono gli italiani contro i vaccini

Si possono però trovare anche esempi, specialmente in testi di scriventi meno consapevoli, in cui il verbo acculturarsi è usato anche con il valore di 'arricchirsi culturalmente, acquisire un grado di cultura più elevato', senza la connotazione negativa vista negli esempi precedenti:

- Nel frattempo il cliente medio si è evoluto, si è acculturato, pretende.

- i miei figli hanno regolarmente frequentato la scuola e ognuno di loro si é laureato, sono tutti dottori, quindi si sono acculturati bene.

Da questo valore del verbo può derivare anche l'uso non connotato negativamente dell'aggettivo derivato dal participio passato evidente negli esempi seguenti, in cui acculturato è impropriamente usato come sinonimo di colto:

- Sei abbastanza acculturato da saper rispondere a queste domande?

- Piú sei acculturato e piú capisci cosa accade attorno a me.

Allo sviluppo del nuovo impiego del verbo acculturarsi con il significato generico di 'acquisire cultura' (prima con accezione negativa poi con un valore più neutro, quasi che il verbo si fosse svincolato dal rapporto semantico con il termine acculturazione) può avere contribuito il fatto che all'aggettivo italiano colto non corrisponde un verbo derivato con il significato 'diventare colto'; è quindi legittimo aspettarsi che il verbo acculturarsi, qualora si ignori il suo rapporto semantico con acculturazione, possa, a partire da cultura, veicolare il significato 'acquisire cultura' in analogia con il modello fornito da verbi quali accalorarsi, addottrinarsi, affaticarsi.

È però opportuno ricordare che, per quanto riconducibile a schemi formativi dellitaliano, il verbo acculturare (attestato a partire dal I974) è entrato a far parte dell'italiano dopo il sostantivo acculturazione (1947), che, stando alle regole di formazione delle parole in italiano dovrebbe esserne un derivato, e che sia il nome sia il verbo sono prestiti dall'inglese, lingua in cui il sostantivo acculturation è attestato a partire dal I88o, quindi, anche in questa lingua, ben prima del verbo to acculturate (I9I7), sebbene il sostantivo sia formalmente un derivato del verbo. Ciò non deve sorprendere, perché nelle terminologie tecniche e specialistiche (formate principalmente da sostantivi) accade spesso che il sostantivo sia più necessario e il verbo corradicale compaia, qualora sia ritenuto utile, solo dopo che il sostantivo si sia stabilizzato.

Da quanto si può ricavare da dizionari quali l'Oxford English Dictionary e il Merriam-Webster, il verbo inglese to acculturate viene usato solo nei valori specifici dell'antropologia e della sociologia, e non ha quindi sviluppato i significati recentemente manifestati dal verbo italiano acculturarsi. La differenza formale tra l'inglese acculturate e l'italiano acculturare è dovuta alla presenza nel verbo inglese del suffisso -ate, che si aggiunge (assieme al prefisso ad- >ac-) al sostantivo di base culture per formare il verbo. Tale suffisso è stato estratto in un periodo non precedente al XVI secolo dalla terminazione che caratterizza il tema del participio passato latino che si ritrova in aggettivi deverbali inglesi di origine latina come separate 'separato', aggravate 'aggravato, peggiorato'. A partire da questi aggettivi sono stati formati per conversione verbi come to separate (cfr. latino sepăro 'dividere, separare') e to aggravate 'aggravare, peggiorare' (cfr. latino aggrāvo 'appesantire, rendere difficile, peggiorare'). La presenza di tale terminazione nei verbi inglesi di origine latina ha favorito l'identificazione di -ate come un suffisso verbalizzatore, che può essere impiegato al posto della più comune conversione per 
formare verbi: cfr. inglese vaccine 'vaccino' > to vaccinate 'vaccinare', assassin 'assassino' > to assassinate 'assassinare'.

In conclusione, mentre l'impiego del verbo acculturarsi nel senso di 'acquisire (o cercare di acquisire) una cultura raccogliticcia, superficiale, spesso velleitaria' è ormai di uso comune, il suo impiego nel senso neutro di 'acquisire un grado di cultura elevato' è da evitare, e tanto meno vi sono motivi per usare l'aggettivo acculturato come sinonimo di colto.

\section{Cita come:}

Claudio lacobini, Colto e acculturato sono sinonimi?, "Italiano digitale", 2021, XVI, 2021/1 (gennaiomarzo)

DOI: $10.35948 / 2532-9006 / 2021.5473$

\section{Copyright 2021 Accademia della Crusca}

Pubblicato con licenza creative commons CC BY-NC-ND 